Design and fabrication of a 3D-structured gold film with nanopores for local electric field enhancement in the pore

This content has been downloaded from IOPscience. Please scroll down to see the full text.

2016 Nanotechnology 27065302

(http://iopscience.iop.org/0957-4484/27/6/065302)

View the table of contents for this issue, or go to the journal homepage for more

Download details:

IP Address: 152.78.130.228

This content was downloaded on 17/02/2016 at $16: 33$

Please note that terms and conditions apply. 


\title{
Design and fabrication of a 3D-structured gold film with nanopores for local electric field enhancement in the pore
}

\author{
James A Grant-Jacob ${ }^{1,4}$, Swe Zin Oo ${ }^{1,2,4}$, Francesca Carpignano ${ }^{1,3}$, \\ Stuart A Boden ${ }^{2}$, William S Brocklesby ${ }^{1}$, Martin D B Charlton ${ }^{2}$ and \\ Tracy Melvin ${ }^{1,2}$ \\ ${ }^{1}$ Optoelectronics Research Centre, University of Southampton, Southampton, SO17 1BJ, UK \\ ${ }^{2}$ School of Electronics and Computer Science, University of Southampton, Southampton, SO17 1BJ, UK \\ ${ }^{3}$ Dipartimento di Ingegneria Industriale e dell'Informazione, University of Pavia, Pavia, I-27100, Italy \\ E-mail: tm@orc.soton.ac.uk
}

Received 20 September 2015, revised 5 November 2015

Accepted for publication 20 November 2015

Published 18 December 2015

\begin{abstract}
Three-dimensionally structured gold membrane films with nanopores of defined, periodic geometries are designed and fabricated to provide the spatially localised enhancement of electric fields by manipulation of the plasmons inside nanopores. Square nanopores of different size and orientation relative to the pyramid are considered for films in aqueous and air environments, which allow for control of the position of electric fields within the structure. Designs suitable for use with $780 \mathrm{~nm}$ light were created. Here, periodic pyramidal cavities produced by potassium hydroxide etching to the $\{111\}$ planes of (100) silicon substrates are used as templates for creating a periodic, pyramidal structured, free-standing thin gold film. Consistent with the findings from the theoretical studies, a nano-sized hole of $50 \mathrm{~nm}$ square was milled through the gold film at a specific location in the cavity to provide electric field control which can subsequently used for enhancement of fluorescence or Raman scattering of molecules in the nanopore.
\end{abstract}

S Online supplementary data available from stacks.iop.org/NANO/27/065302/mmedia

Keywords: nanoscale structures, plasmons, thin films, three-dimensional films, nanoporous, optical properties, template

(Some figures may appear in colour only in the online journal)

\section{Introduction}

Thin films of micro and nanostructured metals are important for the construction of plasmonic devices and microelectromechanical systems (MEMSs). More specifically, twodimensional and three-dimensional gold nanostructures can

${ }^{4}$ These authors have made equal contributions to this work.

Content from this work may be used under the terms of the Creative Commons Attribution 3.0 licence. Any further distribution of this work must maintain attribution to the author(s) and the title of the work, journal citation and DOI. potentially be used to create devices for 'nanofocusing' of plasmons to improve surface-enhanced Raman scattering, (SERS) detection [1]. The fabrication of individual metallic, pyramidal shells as well as ultra-smooth metal films with grooves, bumps, pyramids and holes has previously been demonstrated $[2,3]$, as has direct raster milling with $5 \mathrm{~nm}$ machining precision in $100 \mathrm{~nm}$ thick gold films using a helium ion microscope (HIM) [4]. Nanoporous thin films are of much interest for DNA sequencing applications [5]. As such, routine fabrication of micro and nanostructured thin films is desirable.

Nanopores offer an attractive technology platform for single molecule sensing, notably for polymeric molecules 
such as DNA where high resolution and long read lengths are required for sequencing applications [6-8]. Thus far, three classes of nanopores have been reported: self-assembled, solid state and hybrid nanopores based upon a solid-state framework with a molecular pore [9-11]. Self-assembled nanopores are fabricated with a nanopore embedded in a lipid bilayer, for instance an $\alpha$-haemolysin channel [9]. Solid state nanopores have been created in silicon substrates [12], aluminium oxide substrates [13], high dielectric constant materials (titanium dioxide and hafnium oxide) [14] and graphene sheets [15]. Hybrid nanopores are also well established, such as $\alpha$-haemolysin nanopores within a slightly larger silicon nitride nanopore [16]. Molecules that translocate the nanopore can be detected by electrical or optical approaches [7, 17-19], thus far the optical approaches have been limited to fluorescence methods.

The sensitive direct optical detection of analytes has been achieved by SERS using metallic nanoparticle structures [20], lithographically designed arrays [21] or nanoscale motifs patterned on substrates [22]. In some cases the broad plasmon resonance of the structure is 'tuned' as a function of the shape or separation. Metallic voids offer a better alternative to these structures as photons are coupled into these structures yielding localised plasmon cavities [23] and this provides improved SERS substrates [24]. Using this strategy, some effort has been made to produce substrates suitable for SERS based diagnostic methods through the design of mesostructured metallic materials. This has provided a better understanding as to how the geometry underpins the enhancements. Recently, metallic structure designs have emerged where both localised plasmons as well as a strong coupling to external light is achieved [25-34]. Of these designs, inverted pyramidal substrates (also known as Klarite $($ ) are now well understood with respect to optimal cavity size to achieve the so called 'resonant plasmon cavity' [25, $28,29,35,36]$. The inverted pyramidal structures are created by an anisotropic $\mathrm{KOH}$ etch of (100) silicon wafers with square apertures aligned along the $\langle 100\rangle$ direction. Highly smooth gold surfaces coating over the atomically smooth silicon faces are considered as optimal for creating the 'resonant plasmon cavity', where the polaritons are assumed to oscillate up and down the sides of the inverted pyramid and are reflected by the sharp inverted pyramid edge. We have recently investigated these pyramidal cavities with a view to develop strategies for the sensitive detection and identification of low copy numbers of biomolecules, including DNA. In the course of the investigation, it was established that the detection could be improved by fabricating structures with highly smooth gold of sub-50 nm roughness by e-beam evaporation [37].

Here we investigate for the first time the potential to create a three-dimensional structured and metallic film membrane with nanopores. This is based upon the highly efficient SERS substrate with an inverted pyramid geometry; the chosen design is for Raman interrogation with a $780 \mathrm{~nm}$ operational wavelength with a view to detect single molecules or particles directly in the pore.

\section{Materials and methods}

\subsection{Theoretical design of pyramidal membranes with a nanopore}

Theoretical simulation of the electric field intensity distribution within an illuminated gold periodic pyramidal structured film with a nanopore was evaluated using software suite, RSoft DiffractMOD, Synopsis Inc. The pyramidal cavities are $1.5 \mu \mathrm{m} \times 1.5 \mu \mathrm{m}$ square and $1 \mu \mathrm{m}$ deep with a pitch of $2 \mu \mathrm{m}$. Figure 1 includes illustrations of the inverted pyramid where gold is $100 \mathrm{~nm}$ on the upper surface and $\sim 58 \mathrm{~nm}$ thick on the sidewall (and is estimated from the following: sin(angle of the sidewall) $\times$ thickness on the upper surface $(\mathrm{nm})$ ) and where a square hole is drilled at the base of the pyramidal cavity. The pyramid faces are oriented at an inclination of $\alpha=35.3^{\circ}$ to the normal. Each unit cell (each inverted pyramid), as shown in figure 1(a), is treated as a periodic array and the transmission line formulation for the boundary condition and the $z$-direction is defined as the launch field.

DiffractMOD employs the Rigorous Coupled Wave Analysis method to obtain the backward diffraction efficiency of the surface when illuminated by a plane wave from above (normal to the upper face of the pyramid cavities). A transmission line analysis is applied to an expansion of the refractive index and Electromagnetic field vectors in Fourier space on a cell-by-cell basis, across a fine mesh superimposed on the threedimensional representation of the refractive index.

In our simulation, periodic boundary conditions are used at the sides and number of harmonics is set to ' 7 ' in $x$ and $y$ coordinate transverse to the primary direction, $z$. The number of harmonics is used to expand the refractive index and field in Fourier space resulting in accurate simulation. Simulations are performed for a wide spectral range $(400 \mathrm{~nm}$ to $1500 \mathrm{~nm}$ with $5 \mathrm{~nm}$ steps), typically with TM-polarisation. As a figure of merit, the zero order diffraction efficiency (specular reflection) is obtained and analysed. The plasmonic properties of gold coated pyramidal cavities in silicon substrates have been previously evaluated theoretically [28], but here thin gold films with similarly sized pyramidal cavities with square nanopores in the base (free of the silicon substrate) are considered. Our aim is to establish the impact of the square nanopore (i) dimension and (ii) orientation with respect to the pyramid (either in phase with the pyramid base or at $45^{\circ}$ to it (see figure 2)) and the incident angle of the illumination. In order to obtain a numerical figure of merit for the $E$-field density in and around the drilled square hole, an $E$-field monitor is applied according to equation (1) which integrates the field over a pre-defined volume of space:

$$
U_{E}=\frac{1}{2} \int_{V} \operatorname{Re}[\varepsilon(r)]|E(r)|^{2} \mathrm{~d} V,
$$

where $E(r)$ is the electric field, $\varepsilon(r)$ is the spatially dependent permittivity and the integration is performed over $V$, which is the volume of monitor set by length and height/width.

Figure 1 includes schematics of three-dimensional views of the pyramid cavities used for the theoretical simulations where (a) is where the monitor is placed in the $x z$-axis bisecting 
(a)

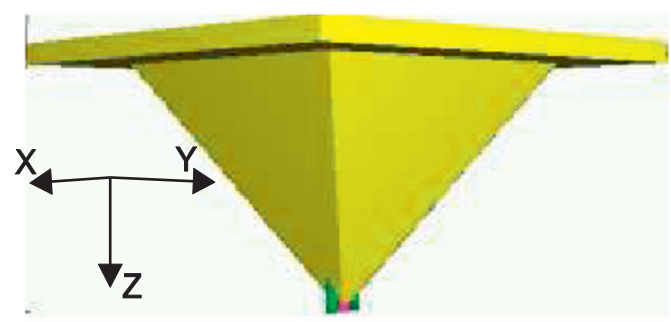

(b)

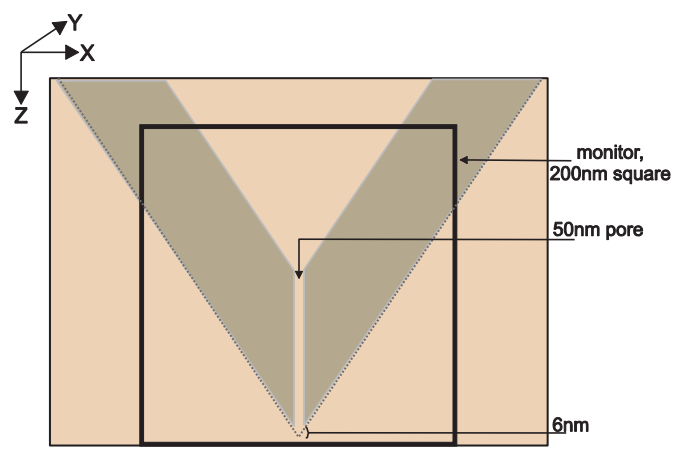

(c)

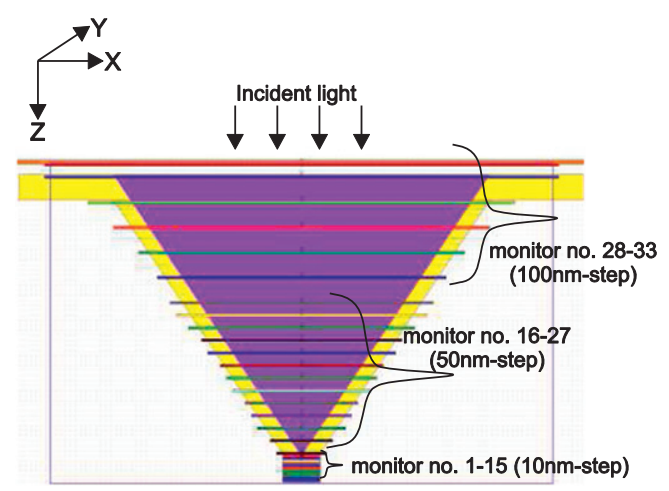

Figure 1. (a) Three-dimensional view of a single unit cell, the gold inverted pyramid, within the periodic gold film (external view) in which the spatial $E$-field monitor (on the $x z$-axis) at the pore is just observable. The nanopore is located at the tip of the inverted pyramid. (b) Larger scale view of the monitor with a $10 \mathrm{~nm}$ nanopore of figure 1 (a). The monitor is $200 \mathrm{~nm}$ square and is placed at $0.221 \mu \mathrm{m}$ below the position of the original tip of the pyramid; the wider nanopores remain within the monitor area. (c) A crosssectional view of a single unit cell in which the spatial $x y$-axis $E$-field monitors are allocated. The cross-sectional view is through the pyramid mid-way along the two opposite sides of the square opening at the top to the tip at the bottom. Fifteen monitors are placed $10 \mathrm{~nm}$ apart through the nanopore in order to observe the detail of changes in the $E$-field distribution across the nanopore (monitors 1-15). The lowest monitor is at $0.121 \mu \mathrm{m}$ below the original tip of the pyramid (before nanopores of different sizes are included). Further $x y$ monitors $50 \mathrm{~nm}$ apart are placed over the inverted pyramid to monitor the $E$-field spatially in the lower part of the cavity (monitors 16-27) and then additional monitors $100 \mathrm{~nm}$ apart to the top of the cavity and above it (monitors 28-33). The monitors are in the $X Y$ plane and the incident light is normal and in the $Z$-direction, as shown by the black arrows. The drawn rectangular box surrounding the outer surface of the cavity is defined as the simulation region for the periodic boundary condition. directly through the nanopore such that the edge of the lower edge of the monitor is $0.121 \mu \mathrm{m}$ below the tip of the pyramid (as if there were no nanopore (note as the pore size increases the central position of the pore opening moves upwards in the $z$-direction, but for our simulations the monitor is always kept in the same relative position for all calculations)). As shown in figure 1(b) the monitor size is $200 \mathrm{~nm}$ square and central in the $x$-direction; the mesh size used is $0.5 \mathrm{~nm}$. Figure 1(c) is a twodimensional view of where the monitors are placed over the inverted whole pyramid cavity and nanopore in the $x y$-axis. The index resolution of the field monitor is set to $1 \mathrm{~nm}$ within the nanopore region. The mesh size is $10 \mathrm{~nm}$.

Figure 2 illustrates how the electric field ( $E$-field) interacts with the inverted pyramid geometry. Figure 2(a) illustrates the convention used here in defining the azimuthal rotation of inverted pyramid and nanopore. The insets of figures 2(b) and (c) are schematic diagrams representing the top view inverted pyramid. The inner square box indicates the orientation of the square nanopore $\left(\phi_{\mathrm{n}}\right)$ at the pyramid base relative to the orientation of the inverted pyramid $\left(\phi_{\mathrm{pyr}}\right)$, shown by the outer square box. For the calculations the key parameters considered include polarisation state, angle of incidence with respect to the surface, azimuth angle with respect to the pyramid sides $\left(\phi_{\mathrm{pyr}}=0^{\circ}\right.$ or $45^{\circ}$ and the azimuth angle of the nanopore $\phi_{\mathrm{n}}=0^{\circ}$ or $45^{\circ}$ in the $x y$-plane (figure 2)).

As illustrated in figure 2(b), for light which is TM-polarised, the orientation of the $E$-field is considered to be parallel to the incident plane and yield charge oscillation between the sidewalls $\mathrm{C}$ and D. Figure 2(c) shows the schematic for the TEpolarised incident illumination; the $E$-field is perpendicular to the incident plane and the charge oscillation occurs across the sidewalls A to B. The arrows indicate the $E$-field orientations. Figure 2(d) illustrates where the charge oscillation is expected to occur when the azimuth angles of the inverted pyramid $\left(\phi_{\text {pyr }}\right)$ and the square nanopore $\left(\phi_{\mathrm{n}}\right)$ are rotated to $0^{\circ}$ and $45^{\circ}$ with respect to the TM-polarised light. The upper and lower part of figure 2(d) illustrates the expected $E$-field orientation for the various orientations of the pyramid and nanopore relative to the illumination (i) $\phi_{\mathrm{pyr}}=0^{\circ}, \phi_{\mathrm{n}}=0^{\circ}$, (ii) $\phi_{\mathrm{pyr}}=0^{\circ}$, $\phi_{\mathrm{n}}=45^{\circ}, \quad$ (iii) $\phi_{\mathrm{pyr}}=45^{\circ}, \quad \phi_{\mathrm{n}}=0^{\circ}, \quad$ (iv) $\phi_{\mathrm{pyr}}=45^{\circ}$, $\phi_{\mathrm{n}}=45^{\circ}$, as considered for the theoretical simulations.

\subsection{Fabrication of pyramidal gold membrane with nanopores}

A silicon substrate is used as a template for creating a pyramidal structured, free-standing gold film. These are fabricated by anisotropic wet etching through square openings in a silicon nitride mask oriented in the $\langle 110\rangle$ direction on a (100) silicon wafer to produce in an array of atomically smooth inverted pyramidal square pits $[25,38]$. The substrate used in this work contains an array of inverted pyramids etched into a $4 \mathrm{~mm} \times 4 \mathrm{~mm}$ square region on the surface with inverted pyramids of $1.5 \mu \mathrm{m} \times 1.5 \mu \mathrm{m}$ square, $1 \mu \mathrm{m}$ deep with a pitch of $2 \mu \mathrm{m}$.

Figure 3 displays a schematic of the fabrication approach for creating the free-standing gold pyramidal film with a 


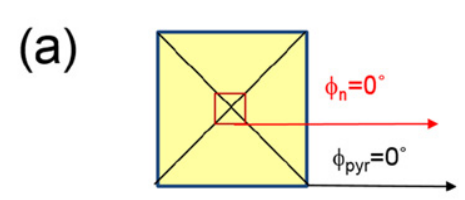

(b)

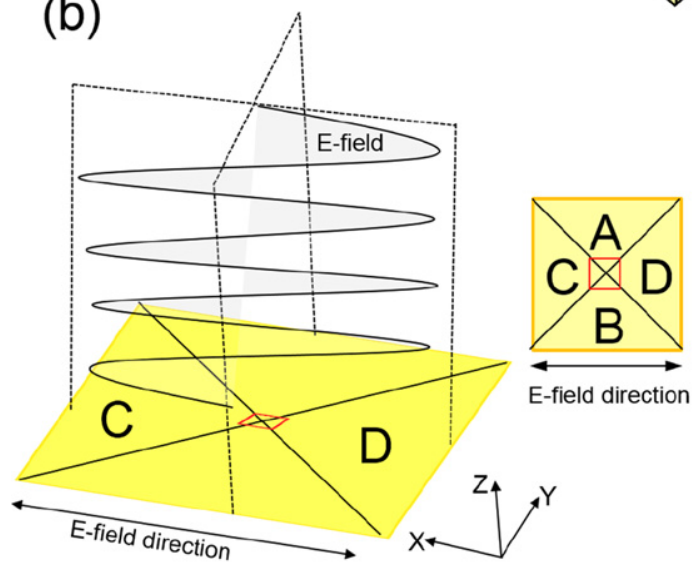

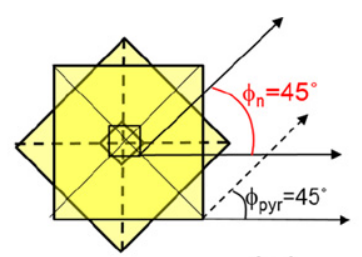

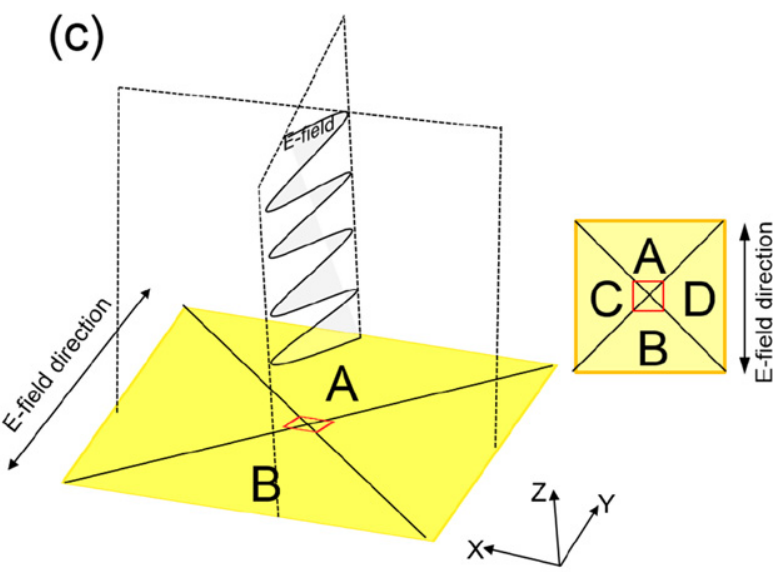

(d)

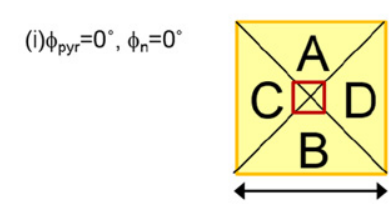

(iii) $\phi_{\text {pyr }}=45^{\circ}, \phi_{\mathrm{n}}=0^{\circ}$

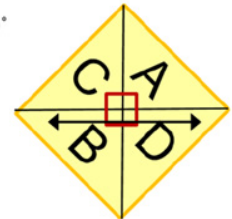

(ii) $\phi_{\text {pyr }}=0^{\circ}, \phi_{\mathrm{n}}=45^{\circ}$

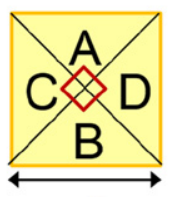

(iv) $\phi_{\text {pyr }}=45^{\circ}, \phi_{n}=45^{\circ}$

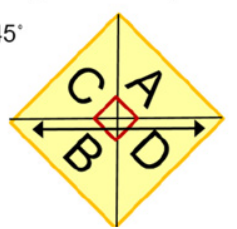

Figure 2. (a) Illustrative drawings of the azimuthal rotation of inverted pyramid $\left(\phi_{\mathrm{pyr}}\right)$ and nanopore $\left(\phi_{\mathrm{n}}\right)$. The first showing the orientation of the azimuth rotation $\left(\phi_{\mathrm{n}}\right.$ and $\left.\phi_{\mathrm{pyr}}=0^{\circ}\right)$ and the second showing $\left(\phi_{\mathrm{n}}\right.$ and $\left.\phi_{\mathrm{pyr}}=45^{\circ}\right)$ (the dashed lines showing $\phi_{\mathrm{n}}$ and $\left.\phi_{\mathrm{pyr}}=0^{\circ}\right)$. The azimuthal/sample rotation is defined by the angle, $\phi_{\mathrm{pyr}}$ for the inverted pyramid and $\phi_{\mathrm{n}}$ for the square nanopore, the rotation of $z$-axis of the sample is with respect to the $x$-axis. (b) and (c) Illustrative drawings of the $E$-field oscillation and direction for the TM and TE modes, with respect to the pyramid cavity orientation. The diagrams are observed from the above the inverted pyramid and the pyramid opening represented as the top face opening with diagonal cross-lines. The schematic shows how each mode is relative to the pyramidal sidewall: (b) TM-polarised incident light; the electric charge oscillates between the sidewalls C and D. (c) TE-polarised incident light; the electric charge oscillates between the sidewalls A and B. (d) Schematic diagrams of the top views of the inverted pyramid with a nanopore as considered theoretically. The arrow indicates the charge oscillation direction for the TM mode.

nanopore. An Edwards E306A Thermal Evaporator is used to coat the silicon templates with a $50 \mathrm{~nm}$ layer of Teflon ${ }^{\circledR}$ and then a $100 \mathrm{~nm}$ layer of gold (see supplementary information for images of the Teflon ${ }^{\circledR}$ and gold coated surfaces). Table 1 displays the experimental parameters used for the thermal deposition of Teflon and gold to achieve the optimal surface smoothness. After removal of the gold coated substrates from the evaporation chamber, $30 \mu \mathrm{l}$ of epoxy is then deposited over the $4 \mathrm{~mm} \times 4 \mathrm{~mm}$ square region on top of the gold pyramid cavities using a pipette. Once the epoxy has cured, the epoxy together with the gold is mechanically separated and lifted from the Teflon $\AA$ coated substrate using a razor blade. The hardened gold-coated epoxy is then placed over a micron-sized aperture (pinhole or gold transmission electron microscope (TEM) grid) and the epoxy dissolved carefully away using acetone. Following removal of the sacrificial epoxy, the gold film was lifted using the pinhole or TEM grid and subsequently inserted into an Agar Scientific, AGG3662, scanning electron microscope (SEM) holder. All SEM imaging in this work was performed using a Carl Zeiss SMT, Inc., Evo® SEM. Imaging and subsequent milling of the pyramidal film at the base of the cavity was achieved using a Carl Zeiss SMT, Inc., Orion ${ }^{\circledR}$ Plus HIM in a similar approach to that previously reported [4]. For milling, the working distance used was $4.5 \mathrm{~mm}$, the beam current was $1 \mathrm{pA}$ and the accelerating voltage was $30 \mathrm{keV}$. The sample was loaded into the HIM load lock and subjected to an 8 min plasma clean with an integrated Evactron remote plasma cleaner (XEI Scientific, Inc.) prior to being transferred to the HIM chamber. This step helps to remove hydrocarbon contamination from the surface, which can interfere with the milling action. A suitable pyramid cavity was first located and centred at a field of view of $900 \mathrm{~nm}$. An image was taken to 


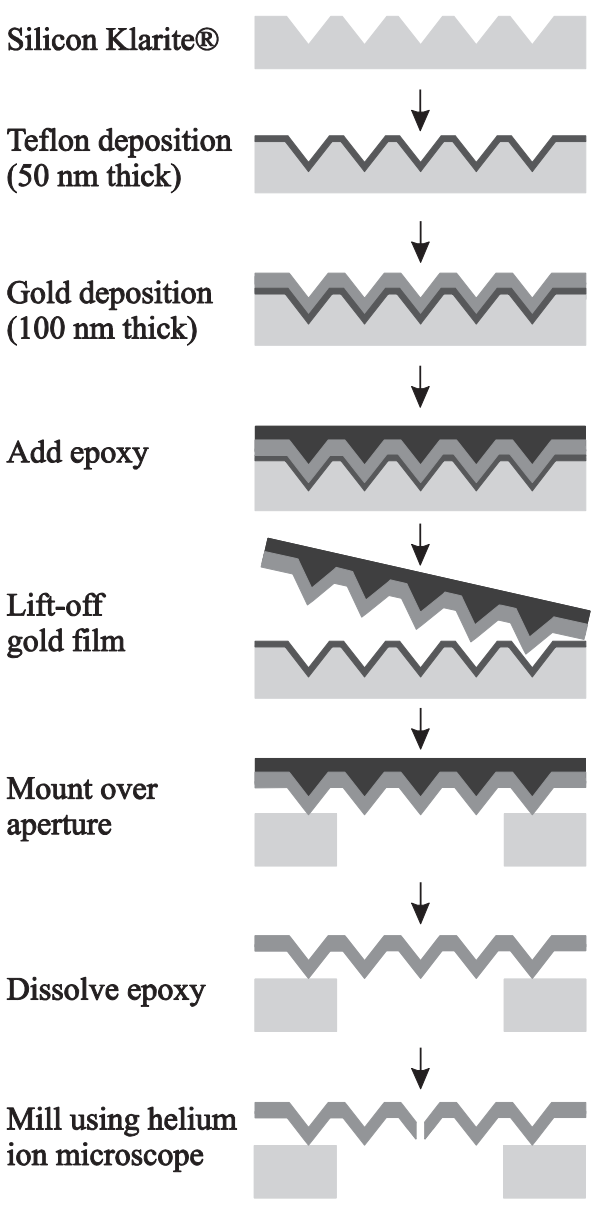

Figure 3. Schematic of the fabrication steps used to produce the freestanding periodic pyramidal nanostructured gold film with a milled nanopore.

record the appearance of the cavity before milling. The beam was then blanked and the field of view decreased (magnification increased $)$ to $100 \mathrm{~nm}(512 \times 512$ pixels $)$. Subscan mode at $50 \%$ field of view was then selected to define a scan area of $50 \mathrm{~nm} \times 50 \mathrm{~nm}(256 \times 256$ pixels $)$. The beam was then raster scanned across this area with a dwell time per pixel of $3 \mu \mathrm{s}$ whilst observing the subscan secondary electron image for evidence of penetration through the film and formation of the pore. Following pore formation, the beam was blanked and the field of view increased (magnification decreased) back to $900 \mathrm{~nm}$. A second image was taken to record the appearance of the fabricated pore using a relatively short dwell time of $10 \mu \mathrm{s}$ to avoid modifying the structure with the imaging beam. HIM was also used to image the surface of the Teflon coated Klarite $₫$ substrate and the free-standing gold film.

\section{Results and discussion}

\subsection{Design of a square nanopore within a 3D-structured pyramidal film}

The nanopore size and orientation within the free-standing 3D-inverted pyramid film was first considered theoretically to gain an understanding of the best configuration to achieve highly localised plasmons trapped inside a nanopore at the base of the pyramidal cavity. The inverted pyramidal pits $\left(54.7^{\circ}\right.$ from the surface plane) are $1.5 \mu \mathrm{m}$ square of $2 \mu \mathrm{m}$ pitch; these are used as a template for the gold membrane. Our rationale for choosing this inverted pyramidal device geometry is the fact that this substrate has previously been shown to be the best geometry for coupling light, showing a strong resonant absorption at $\sim 800 \mathrm{~nm} \mathrm{[24]—at} \mathrm{a} \mathrm{wavelength}$ similar to that used routinely for Raman spectroscopy $(785 \mathrm{~nm})$. As illustrated in figure 1 and as discussed in detail in the materials and methods section, the two-dimensionally periodic gold membrane with a nanopore has a thickness of $100 \mathrm{~nm}$ on the upper surface. The thickness on the sidewall is approximately $58 \mathrm{~nm}$, thus the distance through the gold at the base (tip) of the pyramid is at most $100 \mathrm{~nm}$ (prior to milling). In order to establish the optimal size for a square nanopore in the base of the pyramid cavity to support a highly localised and enhanced $E$-field, theoretical simulations were performed for various sized square nanopores placed at the tip of the inverted pyramid. Square nanopores were evaluated with widths of $10-100 \mathrm{~nm}$ size in $10 \mathrm{~nm}$ incremental steps. Simulations were initially performed with air within the pyramidal cavity and nanopore, then repeated with water inside the pyramids as this medium was considered the most appropriate for future experimental operation in a fluidic system. The refractive index of air is defined as 1 and the refractive index values used for water were varied as a function of wavelength [39].

Figure 4 shows results of preliminary calculations in the form of plots of calculated $E$-field intensity as a function of excitation wavelength at the monitor position within the square nanopore region of the structure. The monitor is $200 \mathrm{~nm}$ square and in the $x z$-direction shown in figures 1(a) and (b), the nanopore to pyramid orientation with respect to the TM-polarised light $\left(\phi_{\mathrm{pyr}}=0^{\circ}, \phi_{\mathrm{n}}=0^{\circ}\right)$ as shown schematically in figure $2(\mathrm{c})$. The monitor bisects the pore and is perpendicular to the side of the pyramid sidewall. Figures 4(a) and (b) show the effect of the size of the square nanopores on the total $E$-field intensity across the area of the monitor for the air, and water environments respectively. The insets show magnified plots for the $E$-field intensity at near infrared wavelengths. This data does not reveal a clear relationship between the localised $E$-field intensity at the pore and the size of nanopore for either background environment (air or water). The $90 \mathrm{~nm}$ sized nanopore provides the highest $E$-field intensity for air or water. We see a clear shift in wavelength between data for air and water, however the spectral shape of the curves remains very similar. This shift is due to the change in refractive index affecting the effective propagation length for the plasmons within the nanopore.

Next, the spatial $E$-field distribution around the nanopore region was investigated whereby the nanopore and inverted pyramid are both at $0^{\circ}$ azimuthal angle (see figure 2(a)) at $780 \mathrm{~nm}$ excitation wavelength. Figure S1 (supplementary information) shows the calculated field distribution across the nanopore region for selected pore sizes (in air and water environments). The $E$-field intensity is mainly confined to the top edge of the pore when the size is $50 \mathrm{~nm}$ (figure 5) and 
Table 1. Conditions used for thermal evaporation of Teflon and gold onto the silicon moulds.

\begin{tabular}{lcccccc}
\hline Material & $\begin{array}{c}\text { Density } \\
\left(\mathrm{g} \mathrm{cm}^{-3}\right)\end{array}$ & Z-value $\left(10^{5} \mathrm{~g} \mathrm{~cm}^{-2} \mathrm{~s}^{-1}\right)$ & Current $(\mathrm{A})$ & $\begin{array}{c}\text { Rate of } \\
\text { deposition }\left(\mathrm{nm} \mathrm{s}^{-1}\right)\end{array}$ & $\begin{array}{c}\text { Chamber } \\
\text { Thickness }(\mathrm{nm})\end{array}$ & \begin{tabular}{c} 
pressure (mbar) \\
\hline Teflon
\end{tabular} \\
Gold & 2.7 & 8.2 & 0.9 & 0.07 & 50 & $1 \times 10^{-6}$ \\
\hline
\end{tabular}

(a)

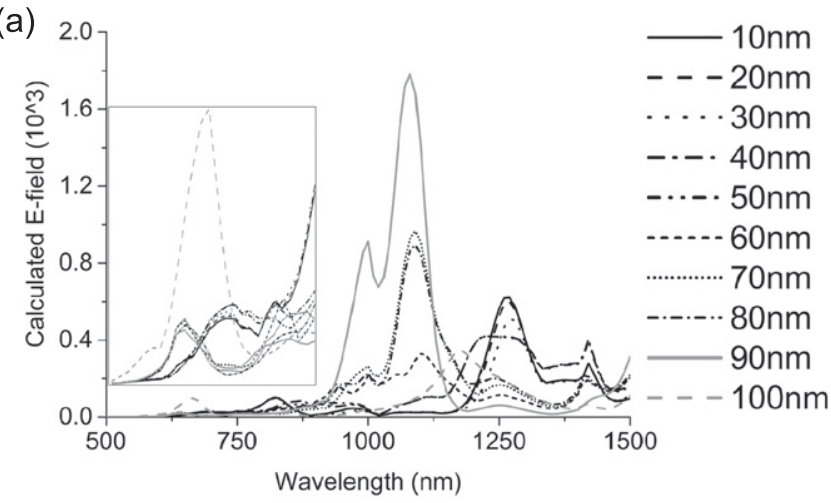

(b)

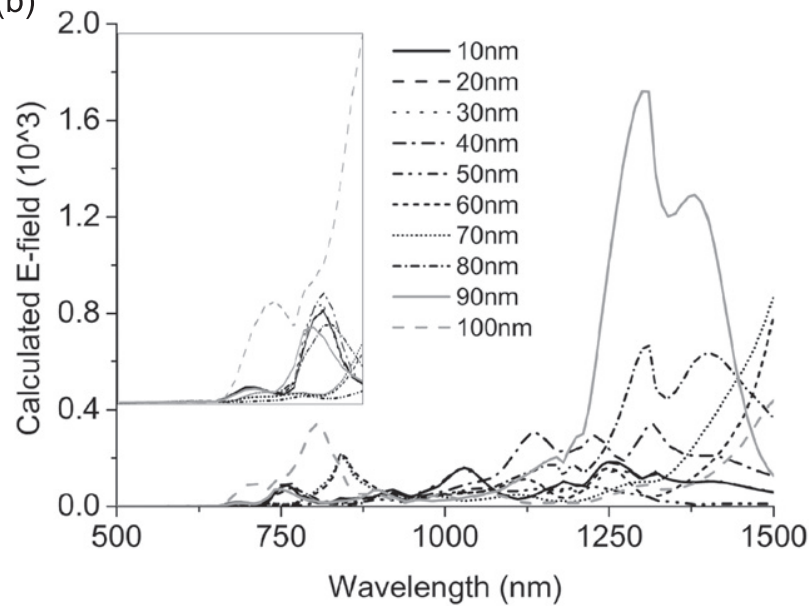

Figure 4. Plots of the total integral of calculated $E$-field across the nanopore as a function of excitation wavelength $(400 \mathrm{~nm}-1500 \mathrm{~nm})$ for different sizes of square nanopore in (a) air and (b) water environments. The size of the nanopore varies from $10 \mathrm{~nm}$ to $100 \mathrm{~nm}$ as indicated by the insert. The inset plots are magnified intensities (by 10 times) for the near infrared region (to scale with the $x$-axis).

smaller (figure S1) (as indicated by the sharp horizontal features of the $E$-field intensity) for the samples in air. Whereas for the inverted pyramidal gold film, in a water environment with a $50 \mathrm{~nm}$ pore size, the $E$-field is highly confined inside the walls of the nanopore. Since the objective here is to develop a system whereby nano-sized species are to be interrogated optically in water inside the pore, the $50 \mathrm{~nm}$ pore is considered optimal.

Other wavelengths of light were considered for our theoretical simulation studies; a video file of the $E$-field distribution within the structure as a function of excitation wavelength for the $50 \mathrm{~nm}$ pore size in the water medium is provided as supplementary information (video 1 ) - where the $E$-field intensity is normalised (with respect to the input energy). In brief (and as shown in figure 6 for $400 \mathrm{~nm}$,

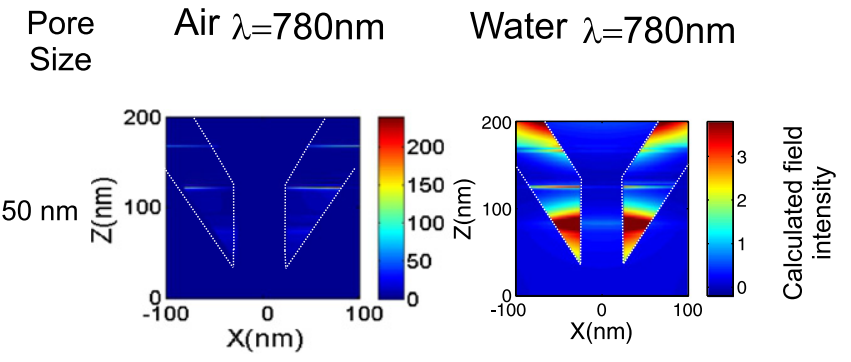

Figure 5. Calculated spatial field distribution of the cross section of the nanopore at $780 \mathrm{~nm}$ operation wavelength. Fields are shown for the $50 \mathrm{~nm}$ nanopore size for both environments; air and water. The white dotted lines are visual guides for the nanopore and inverted pyramid. The resolution of the monitor is $0.5 \mathrm{~nm}$.

$680 \mathrm{~nm} 930 \mathrm{~nm}$ and $1200 \mathrm{~nm}$ ), the $E$-field does not penetrate the nanopore for illumination wavelengths of $400 \mathrm{~nm}$ to $670 \mathrm{~nm}$. The $E$-field intensity and position in the nanopore varies for the excitation wavelengths from $680 \mathrm{~nm}$ to $1390 \mathrm{~nm}$. Even though the $E$-field intensity within the nanopore illuminated at wavelengths up to and around $930 \mathrm{~nm}$ the $E$-field intensity distribution is more 'tightly' localised for $780 \mathrm{~nm}$. The total $E$-field intensity in the pore illuminated with $1200 \mathrm{~nm}$ light is $\sim 14$ times higher than for $780 \mathrm{~nm}$. Figure 5 shows the high spatial localisation of the $E$-field across the middle of the $50 \mathrm{~nm}$ pore in water when illuminated with $780 \mathrm{~nm}$, the $E$-field inside the pore is just observed; thus single molecules passing this intra-nanopore region would experience higher $E$-fields during transit.

The $E$-field intensity and distribution arising from the geometry of the inverted pyramid to nanopore illuminated with polarised light were next investigated. Figure 2(a) provides schematics for the azimuth angle of the pyramid $\left(\phi_{\mathrm{pyr}}\right)$ and nanopore $\left(\phi_{\mathrm{n}}\right)$ the arrows shown provide the orientation of the TM mode of the incident light. First the azimuth angle $\left(\phi_{\text {pyr }}\right)$ is set at $0^{\circ}$ or $45^{\circ}$ which (according to our results $[25,28,29])$ corresponds to the critical angle of polarisation conversion in the inverted pyramid, but in this case where the structure is a pyramidal film with a nanopore of $50 \mathrm{~nm}$ square. Figure 7(a) is a plot of the total normalised $E$-field intensity integrated across the nanopore region (corresponding to the monitor area shown in figures 1(a) and (b)) as a function of wavelength. The total $E$-field inside the nanopore (normalised against the same input energy for each wavelength) shows a significant dependence with wavelength $(400 \mathrm{~nm}$ to $1500 \mathrm{~nm})$ for $\phi_{\mathrm{pyr}}=0^{\circ}$, whereas when $\phi_{\mathrm{pyr}}=45^{\circ}$ (seen in figure 7(b)) it is independent of wavelength. Thus changing the pyramid azimuth angle $\left(\phi_{\text {pyr }}\right)$ in relation to the square pore edges has a large impact on total $E$-field intensity. 

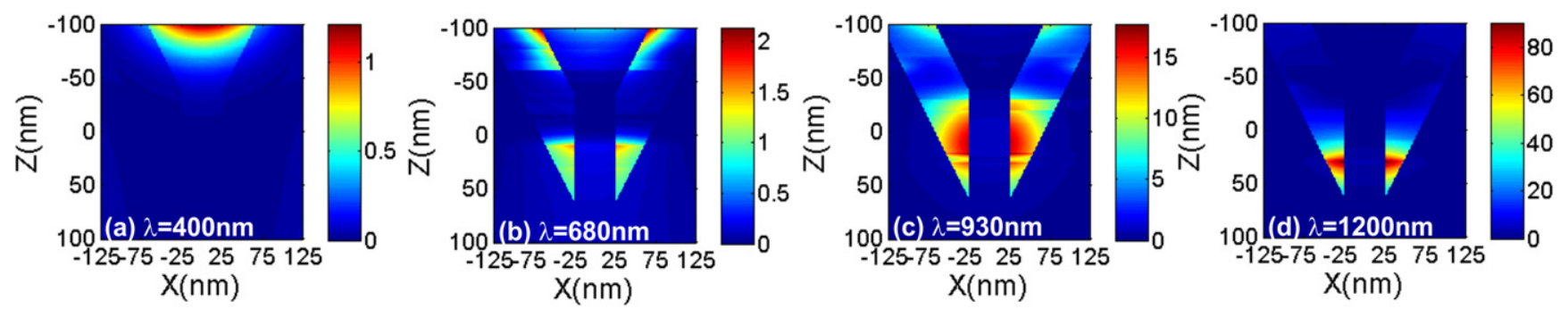

Figure 6. Calculated spatial field distribution of the cross section of the nanopore in water at (a) $400 \mathrm{~nm}$, (b) $680 \mathrm{~nm}$, (c) $930 \mathrm{~nm}$, (d) $1200 \mathrm{~nm}$. (For $780 \mathrm{~nm}$ see figure 5.) (All data shown are normalised against the input energy.) Note that cross-sectional plots of the $E$-field intensity across the nanopore in water are also shown in video 2 (supplementary information) as a function of position from monitor 1 (figure 1(c)). The size and resolution of the monitor are $250 \times 200 \mathrm{~nm}$ and $1 \mathrm{~nm}$, respectively.

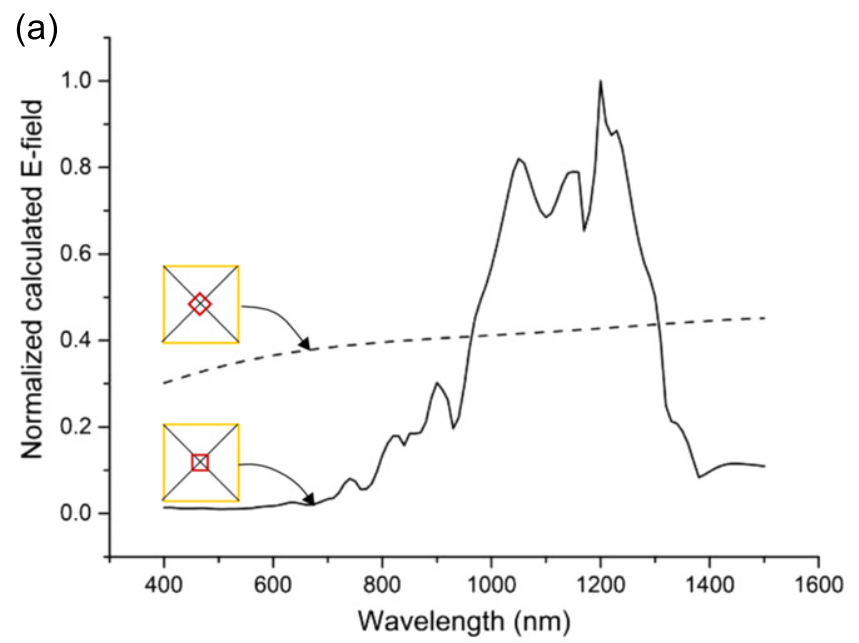

(b)

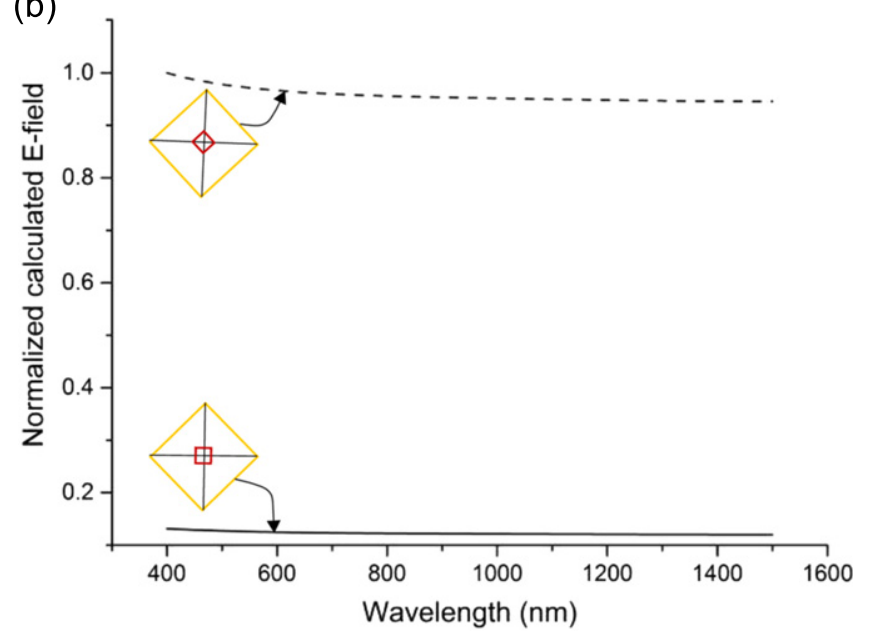

Figure 7. (a) Normalised $E$-field in the nanopore for monitor shown in figures 1(a) and (b) as a function of wavelength but where the orientation of the inverted pyramid and square nanopore are different (see figure 2(d) for azimuth angle definitions). Solid line is for the nanopore and inverted pyramid where $\phi_{\mathrm{pyr}}=0^{\circ}, \phi_{\mathrm{n}}=0^{\circ}$, the dashed line is for $\phi_{\mathrm{pyr}}=0^{\circ}, \phi_{\mathrm{n}}=45^{\circ}$. Figure 7(b) Solid line is for the nanopore and inverted pyramid where $\phi_{\mathrm{pyr}}=45^{\circ}, \phi_{\mathrm{n}}=0^{\circ}$, the dashed line is for $\phi_{\mathrm{pyr}}=45^{\circ}, \phi_{\mathrm{n}}=45^{\circ}$.

At this juncture, it is important to look in detail at the spatial distribution of the $E$-field within the nanopore illuminated with $780 \mathrm{~nm}$ light. Figure 8 shows $E$-field distribution for a $50 \mathrm{~nm}$ square nanopore illuminated with $780 \mathrm{~nm}$ light, monitored from the top of the nanopore in the $x y$-axis as well as in the $x z$-axis. The results suggest that the $E$-field distribution across the nanopore is uniform over the upper four faces of the pyramid in the vicinity of the nanopore, but for the case where the square nanopore is out of phase with the upper pyramid edge $\left(\phi_{\mathrm{pyr}}=0^{\circ}, \phi_{\mathrm{n}}=45^{\circ}\right)$ or $\left(\phi_{\mathrm{pyr}}=45^{\circ}\right.$ ,$\left.\phi_{\mathrm{n}}=0^{\circ}\right)$ there is no penetration of the $E$-field into the nanopore. However the $E$-field penetrates the nanopore for the cases where the square nanopore edges are in phase with the upper pyramid edges: for the case $\left(\phi_{\mathrm{pyr}}=0^{\circ}, \phi_{\mathrm{n}}=0^{\circ}\right)$ the $E$-field is localised spatially within the pore. And for the case $\left(\phi_{\mathrm{pyr}}=45^{\circ}, \phi_{\mathrm{n}}=45^{\circ}\right)$, the $E$-field penetrates the pore, but is less localised. Thus the theoretical study provides evidence that the incident plane of illuminating light plays a significant role in determining the $E$-field intensity inside the nanopore. This is not so surprising since surface plasmon polaritons are assumed to oscillate up and down the sides of the inverted pyramid and will be reflected by the sharp top edges of the inverted pyramid and square sides of the nanopore at the base [25]. However, when the nanopore is in phase with the pyramid and the polarisation plane of the incident light is symmetrical to the faces of the pyramid cavity as well as the nanopore square edges, there is a strong dependence of $E$-field as a function of wavelength inside the nanopore (figure 7).

The $E$-field intensity across the width of the pyramid was analysed using higher resolution spatial monitors at a range of height positions respective to base of the pyramid as depicted in figure 1(c). The $E$-field intensity for the structure where $\left(\phi_{\mathrm{pyr}}=0^{\circ}, \phi_{\mathrm{n}}=45^{\circ}\right)$ and the incident light is $780 \mathrm{~nm}$ varies as a function of Z-length (height of the device) and is shown in the plot displayed in figure S3 (supplementary information). The $E$-field intensity inside the cavity of the pyramid is much higher ( $\sim 9$ times) compared to the field inside the nanopore; however, this high field intensity occurs at the sidewall of the pyramid over a much larger surface area. Video 2 (supplementary information) shows the $E$-field intensity plots for all the monitors over the whole structure. The maximum total $E$-field intensity occurs $860 \mathrm{~nm}$ above the tip of the pyramid and is confined to the sidewalls as shown in figure S3(a). Whilst the $E$-field intensity is greatest on the pyramid sidewalls $860 \mathrm{~nm}$ above the nanopore, it is anticipated that the majority of the molecules traversing the pore will experience the $E$-field within the nanopore only. 
(i)
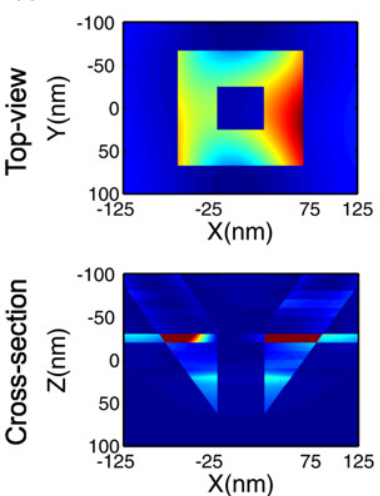

(iii)
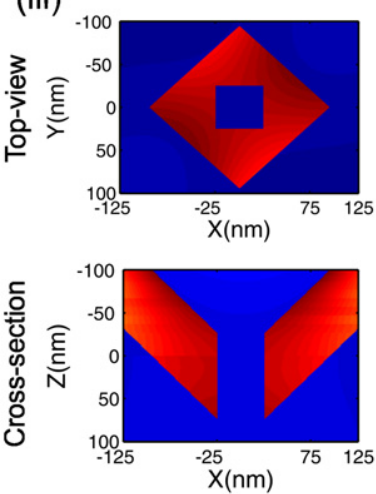

(ii)

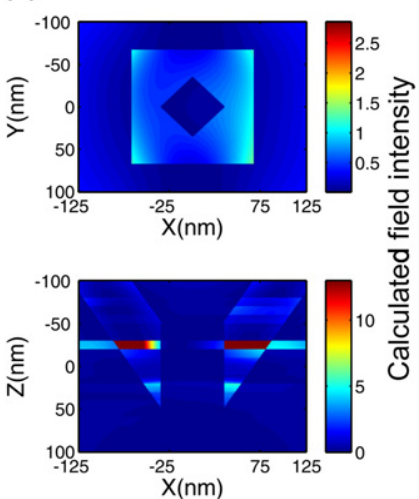

(iv)
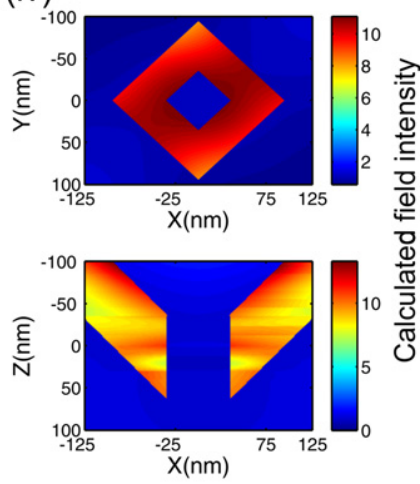

Figure 8. Calculated field intensity plots for the top of the nanopore (the $x y$-monitor 15 (figure 1(c)), and directly below, the crosssectional view $x z$ monitor (figures $1(a)$ and (b)), with $780 \mathrm{~nm}$ illumination in a water environment, where the orientation of the inverted pyramid and square nanopore are different (i) $\phi_{\mathrm{pyr}}=0^{\circ}$, $\phi_{\mathrm{n}}=0^{\circ}$ (see figure 2(d)); (ii) $\phi_{\mathrm{pyr}}=0^{\circ}, \phi_{\mathrm{n}}=45^{\circ}$; (iii) $\phi_{\mathrm{pyr}}=45^{\circ}$, $\phi_{\mathrm{n}}=0^{\circ}$; (iv) $\phi_{\mathrm{pyr}}=45^{\circ}, \phi_{\mathrm{n}}=45^{\circ}$. The size and resolution of the monitor are $250 \times 200 \mathrm{~nm}$ and $1 \mathrm{~nm}$, respectively.

\subsection{Characterisation of the pyramidal gold membrane with nanopores}

The fabrication of a three-dimensional gold membrane film using the template method demonstrated here is novel. Figure 9 displays a SEM image of a $100 \mathrm{~nm}$ thick, freestanding gold film containing an array of nano-sized gold pyramids suspended over a TEM grid. The TEM grid is $3.05 \mathrm{~mm}$ in diameter and contains 19 square apertures per millimetre. Each square aperture is $\sim 25 \mu \mathrm{m}$ wide and on a $\sim 53 \mu \mathrm{m}$ pitch. From additional SEM imaging, it is evident that the pyramidal film remains intact over a region of approximately $6 \mathrm{~mm}^{2}$. Visible from the images are residual pyramids scattered over the surface of the TEM grid. These are likely the result of tears in the gold film allowing the pyramids be left on the TEM grid during lift-off of the gold film.

To evaluate the pyramid roughness and structure in more detail, the film was imaged using a Carl Zeiss SMT, Inc., Orion ${ }^{\circledR}$ Plus HIM. Additional images are shown in the supplementary information (figures S4-S6). These additional evaluations show that the grain structure of the thermally

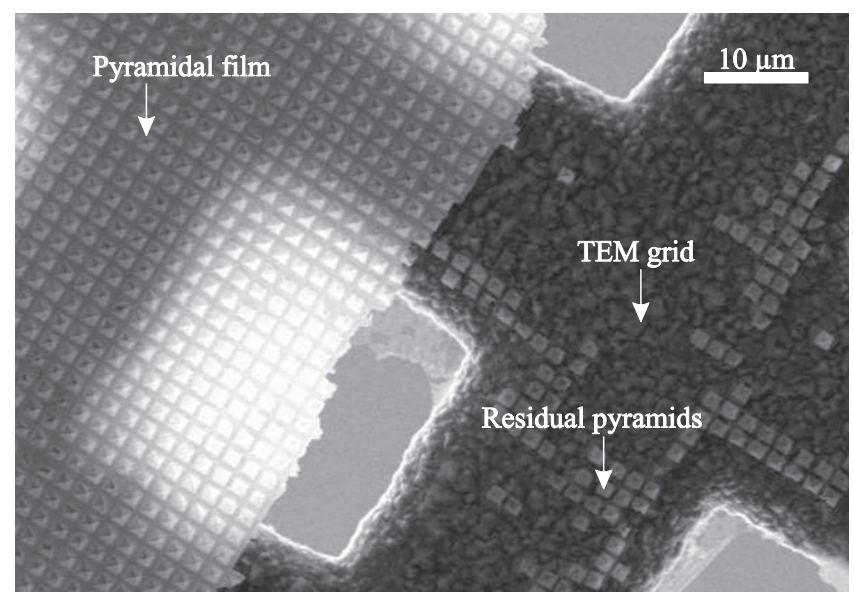

Figure 9. Scanning electron micrograph of edge of the gold pyramidal film suspended over a TEM grid. The pyramid cavities are on the topside of the film. The film is suspended over the region illustrated in the left side of the film; the residual pyramids are from an area of the film where the full structure was not 'lifted off' fully from the silicon mould.

evaporated gold as visible from the HIM images in figures 10 and figure S5 occurs from the evaporated gold. The average size of each grain of gold is $\sim 40 \mathrm{~nm}$. Optimised evaporation of gold on pyramid cavities have previously been reported by us [40]. Although there appears to be periodicity in the evaporated gold film shown in figures 10 and figure S5, by taking a single pixel slice across one of the pyramids (one face to another) as shown in figure S6(a), it is evident that the distribution of the gold particles is stochastic, as confirmed by figure S6(b), the Fourier transform of the data from figure S6 (a), showing that there is little periodicity. To verify that defects in the Teflon deposition on the silicon surface did not contribute to the observed roughness of the gold, a HIM image of the Teflon coated inverted pyramids was taken (see figure S4, supplementary information). The surface of the Teflon is relatively smooth compared with the gold surface (figure S5), therefore the small gold clusters seen are similar in size to those seen by others for ultra-smooth gold [41]. Whilst we consider that the surface roughness may have an impact on the $E$-field intensity, experimental data reported for the inverted pyramidal structures suggest that the inverted pyramidal structure out-performs planar structures, notably in coupling the light (with $\sim 70 \%$ absorption), to provide enhanced levels of detection $[25,29,42]$. Indeed our own studies suggest that the intensity of the Raman spectrum obtained for thermally evapourated and e-beam deposited gold inverted pyramid structures of the geometry here, are optimal when the gold is deposited by e-beam methods [37]. However the impact of surface roughness should not be discounted, as demonstrated by others where gold motifs of lines $(\sim 10 \mathrm{~nm}$ wide) were investigated [43]. The studies suggest that the SERS effect can be more pronounced in a spectral region far from the localised surface plasmon resonance with nanoscale surface roughness of the gold within the device [42]. 
(a)

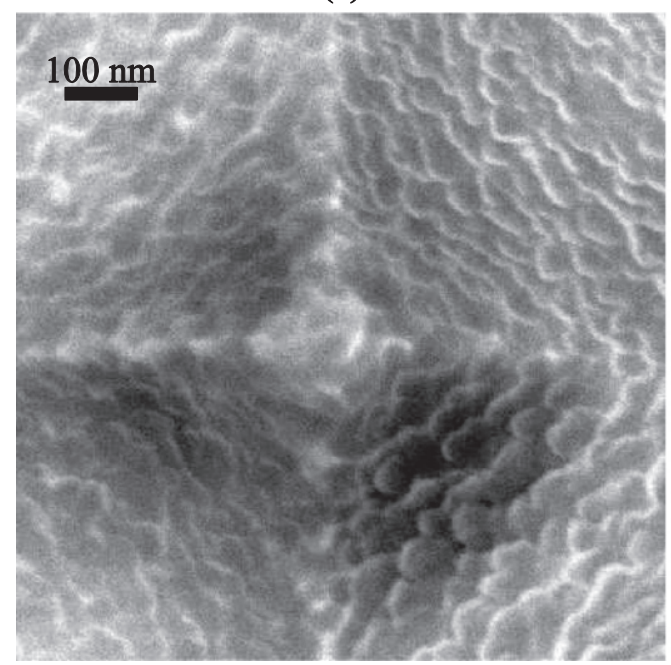

(b)

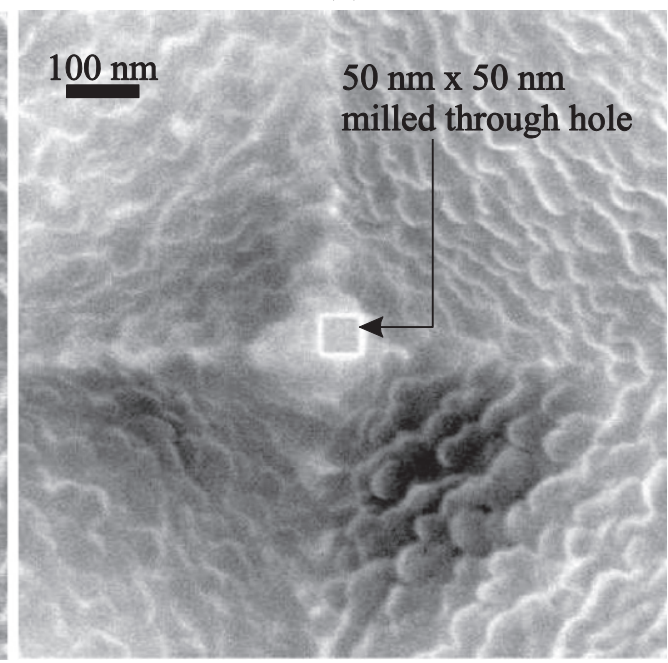

Figure 10. Helium ion microscope image of a single pyramid (a) before and (b) after milling a $50 \mathrm{~nm}$ square hole at $45^{\circ}$ to the pyramid top opening. The nanopore is visible in the base of the cavity - as indicated by the black arrow. In this image the pyramid cavities are projecting downward.

\subsection{Nanopore milling}

Using the HIM, a $50 \mathrm{~nm} \times 50 \mathrm{~nm}$ square hole was milled though the suspended film using a similar milling technique that was employed by Scipioni et al [4]. Figure 10 shows HIM images of a single pyramid before (figure 10(a)) and after milling (figure 10(b)) the $50 \mathrm{~nm} \times 50 \mathrm{~nm}$ hole through the base of a pyramid in free-standing gold. The milling process took approximately $30 \mathrm{~s}$ to complete, with penetration through the base of the pyramid being detected as a sudden decrease in the secondary emission from the area being scanned. As reported previously [4], it was possible to observe the hole during machining, allowing the milling to be stopped when the hole had widened sufficiently to cover the entire scan area. The border of the milled square hole is visible at the centre of the image in figure $10(\mathrm{~b})$ as a square white line. This white line in the image (figure 10(b)) is the result of secondary emission from the sharp edges of the square hole. The milled hole is measured to be $50 \pm 2 \mathrm{~nm}$ wide and the radius of curvature of each corner is approximately $4 \mathrm{~nm}-6 \mathrm{~nm}$. Its centre is offset from the apex of the pyramid by $\sim 15 \mathrm{~nm}$ in the $x$-axis and $\sim 35 \mathrm{~nm}$ in the $y$-axis. Although the more conventional gallium focused ion beam technique can be used to mill pores with $50 \mathrm{~nm}$ dimensions, the smaller probe size of the HIM leads to more precise milling, enabling a smaller radius of curvature at the corners of the square feature and so a pore shape more closely resembling the simulated structure.

\section{Conclusion}

These studies provide the first example for the design and fabrication of a nanopore in the base of three-dimensionally structured inverted pyramidal gold film. The design has been optimised here for the use with $780 \mathrm{~nm}$ light where pyramidal cavities of $1.5 \mu \mathrm{m} \times 1.5 \mu \mathrm{m}$ square and $1 \mu \mathrm{m}$ deep with a pitch of $2 \mu \mathrm{m}$ with a $50 \mathrm{~nm}$ square pore. The effect of the azimuthal rotation of (i) the inverted pyramid and (ii) the nanopore on the localised field intensity within the pore under illumination with linearly polarised light was examined. It was observed that the azimuth angle of the inverted pyramid has more impact on the localised field intensity in the pore as compared to the azimuth angle of the square nanopore. The spatial $E$-field intensity and distribution across the nanopore was considered in detail for the $50 \mathrm{~nm}$ pore when the inverted pyramid and nanopore are both with an azimuth angle of $0^{\circ}$, where the $E$-field intensity penetrates the pore, providing a uniform $E$-field density through which an analyte could be interrogated during transit through the pore.

Building upon the theoretical studies, we have also demonstrated for the first time the successful fabrication of a free-standing $100 \mathrm{~nm}$ thick gold film of arrays of pyramids, using a template method. In addition, we have showed the milling of a $50 \mathrm{~nm} \times 50 \mathrm{~nm}$ square hole through the base of a free-standing gold film pyramid. We envisage that this method of thin film fabrication can be extended to other devices with other geometries for analytical applications where detection is facilitated by plasmon-enhancement [3], as well as for the construction of metallic MEMS and nanoelectro-mechanical systems [44].

\section{Acknowledgments}

This work was supported by the BBSRC (Ref: BB/I023720/ 1 and BB/I022791/1) and the CARIPLO Foundation for funding FC. We are very grateful to Renishaw Diagnostics for providing Klarite ${ }^{\circledR}$ samples. 


\section{References}

[1] Barnes W L, Dereux A and Ebbesen T W 2003 Surface plasmon subwavelength optics Nature 424 824-30

[2] Xu Q, Tonks I, Fuerstman M J, Love J C and Whitesides G M 2004 Fabrication of free-standing metallic pyramidal shells Nano Lett. 4 2509-11

[3] Nagpal P A L, Nathan C, Oh S-H and Norris D J 2009 Ultrasmooth patterned metals for plasmonics and metamaterials Science 325 594-7

[4] Scipioni L, Ferranti D C, Smentkowski V S and Potyrailo R A 2010 Fabrication and initial characterization of ultrahigh aspect ratio vias in gold using the helium ion microscope J. Vac. Sci. Technol. B 28 C6P18-6P23

[5] Branton D et al 2008 The potential and challenges of nanopore sequencing Nat. Biotechnol. 26 1146-53

[6] Wanunu M, Morrison W, Rabin Y, Grosberg A Y and Meller A 2010 Electrostatic focusing of unlabelled DNA into nanoscale pores using a salt gradient Nat. Nanotechnol. 5 160-5

[7] McNally B, Singer A, Yu Z, Sun Y, Weng Z and Meller A 2010 Optical recognition of converted DNA nucleotides for single-molecule DNA sequencing using nanopore arrays Nano Lett. 10 2237-44

[8] Soni G V, Singer A, Yu Z, Sun Y, McNally B and Meller A 2010 Synchronous optical and electrical detection of biomolecules traversing through solid-state nanopores Rev. Sci. Instrum. 81014301

[9] Kasianowicz J J, Brandin E, Branton D and Deamer D W 1996 Characterization of individual polynucleotide molecules using a membrane channel Proc. Natl Acad. Sci. 93 13770-3

[10] Butler T Z, Pavlenok M, Derrington I M, Niederweis M and Gundlach J H 2008 Single-molecule DNA detection with an engineered MspA protein nanopore Proc. Natl Acad. Sci. 105 20647-52

[11] Wendell D, Jing P, Geng J, Subramaniam V, Lee T J, Montemagno C and Guo P 2009 Translocation of doublestranded DNA through membrane-adapted phi29 motor protein nanopores Nat. Nanotechnol. 4 765-72

[12] van den Hout M, Hall A R, Wu M Y, Zandbergen H W, Dekker C and Dekker N H 2010 Controlling nanopore size, shape and stability Nanotechnology 21115304

[13] Chen P, Mitsui T, Farmer D B, Golovchenko J, Gordon R G and Branton D 2004 Atomic layer deposition to fine-tune the surface properties and diameters of fabricated nanopores Nano Lett. 4 1333-7

[14] Knez M, Nielsch K and Niinistö L 2007 Synthesis and surface engineering of complex nanostructures by atomic layer deposition Adv. Mater. 19 3425-38

[15] Venkatesan B M, Estrada D, Banerjee S, Jin X, Dorgan V E, Bae M-H, Aluru N R, Pop E and Bashir R 2012 Stacked graphene- $\mathrm{Al}_{2} \mathrm{O}_{3}$ nanopore sensors for sensitive detection of DNA and DNA-protein complexes ACS Nano 6 $441-50$

[16] Hall A R, Scott A, Rotem D, Mehta K K, Bayley H and Dekker C 2010 Hybrid pore formation by directed insertion of [alpha]-haemolysin into solid-state nanopores Nat. Nanotechnol. 5 874-7

[17] Nicoli F, Verschueren D, Klein M, Dekker C and Jonsson M P 2014 DNA translocations through solid-state plasmonic nanopores Nano Lett. 14 6917-25

[18] Benner S, Chen R J, Wilson N A, Abu-Shumays R, Hurt N, Lieberman K R, Deamer D W, Dunbar W B and Akeson M 2007 Sequence-specific detection of individual DNA polymerase complexes in real time using a nanopore $\mathrm{Nat}$. Nanotechnol. 2 718-24
[19] Lieberman K R, Cherf G M, Doody M J, Olasagasti F, Kolodji Y and Akeson M 2010 Processive replication of single DNA molecules in a nanopore catalyzed by phi29 DNA polymerase J. Am. Chem. Soc. 132 17961-72

[20] Barhoumi A and Halas N J 2011 Detecting chemically modified DNA bases using surface-enhanced Raman spectroscopy J. Phys. Chem. Lett. 2 3118-23

[21] Camden J P, Dieringer J A, Zhao J and Van Duyne R P 2008 Controlled plasmonic nanostructures for surface-enhanced spectroscopy and sensing Acc. Chem. Res. 41 1653-61

[22] Green M, Liu F M, Cohen L, Kollensperger P and Cass T 2006 SERS platforms for high density DNA arrays Faraday Discuss. 132 269-80

[23] Fang N, Lee H, Sun C and Zhang X 2005 Sub-diffractionlimited optical imaging with a silver superlens Science $\mathbf{3 0 8}$ $534-7$

[24] Mahajan S, Richardson J, Brown T and Bartlett P N 2008 SERS-melting: a new method for discriminating mutations in DNA sequences $J$. Am. Chem. Soc. 130 15589-601

[25] Perney N M B, Baumberg J J, Zoorob M E, Charlton M D B, Mahnkopf S and Netti C M 2006 Tuning localized plasmons in nanostructured substrates for surface- enhanced Raman scattering Opt. Express 14 847-57

[26] Muttalib M F A, Oo S Z and Charlton M D B 2012 Integrated Optics: Devices, Materials, and Technologies XVI ed $\mathrm{J}$ E Broquin and $\mathrm{G} \mathrm{N}$ Conti

[27] Miao X, Wilson B K and Lin L Y 2008 Localized surface plasmon assisted microfluidic mixing Appl. Phys. Lett. 92 124108

[28] Oo S Z, Charlton M D B, Pollard M E, Pearce S J and Chen R Y 2012 3D analysis of surface plasmon dispersion for SERS sensor based on inverted pyramid nanostructures Proc. SPIE 8269 82691Y

[29] Perney N M B, de Abajo F J G, Baumberg J J, Tang A, Netti M C, Charlton M D B and Zoorob M E 2007 Tuning localized plasmon cavities for optimized surface-enhanced Raman scattering Phys. Rev. B 76035426

[30] Sigle D O, Perkins E, Baumberg J J and Mahajan S 2013 Reproducible deep-UV SERRS on aluminum nanovoids J. Phys. Chem. Lett. 4 1449-52

[31] Johnson R P, Mahajan S, Abdelsalam M E, Cole R M, Baumberg J J, Russell A E and Bartlett P N 2011 SERS from two-tier sphere segment void substrates Phys. Chem. Chem. Phys. 13 16661-5

[32] Speed J D, Johnson R P, Hugall J T, Lal N N, Bartlett P N, Baumberg J J and Russell A E 2011 SERS from molecules bridging the gap of particle-in-cavity structures Chem. Commun. 47 6335-7

[33] Cui L, Mahajan S, Cole R M, Soares B, Bartlett P N, Baumberg J J, Hayward I P, Ren B, Russell A E and Tian Z Q 2009 UV SERS at well ordered Pd sphere segment void (SSV) nanostructures Phys. Chem. Chem. Phys. 11 1023-6

[34] Kelf T A, Sugawara Y, Cole R M, Baumberg J J, Abdelsalam M E, Cintra S, Mahajan S, Russell A E and Bartlett P N 2006 Localized and delocalized plasmons in metallic nanovoids Phys. Rev. B 74245415

[35] Oo S Z, Chen R Y, Siitonen S, Kontturi V, Eustace D A, Tuominen J, Aikio S and Charlton M D B 2013 Disposable plasmonic plastic SERS sensor Opt. Express 21 18484-91

[36] Oo S Z, Charlton M D B, Eustace D, Chen R Y, Pearce S J and Pollard M E 2012 Optimization of SERS enhancement from nanostructured metallic substrate based on arrays of inverted rectangular pyramids and investigation of effect of lattice non-symmetry Proc. SPIE 8234 823406-7

[37] Carpignano F, Grant-Jacob J A, Lamb J, Pechstedt K, Brocklesby W S and Melvin T 2014 Direct detection of DNA on gold structured planar substrates by Raman 
microscopy 2014 Fotonica AEIT Italian Conf. on Photonics Technologies (12-14 May 2014) p 1-4

[38] Sato K, Shikida M, Yamashiro T, Tsunekawa M and Ito S 1999 Roughening of single-crystal silicon surface etched by $\mathrm{KOH}$ water solution Sensors Actuators A 73 122-30

[39] Palik E D (ed) 1991 Handbook of Optical Constants of Solids II (Boston, MA: Academic)

[40] Kuttge M, Vesseur E J R, Verhoeven J, Lezec H J, Atwater H A and Polman A 2008 Loss mechanisms of surface plasmon polaritons on gold probed by cathodoluminescence imaging spectroscopy Appl. Phys. Lett. 93113110
[41] Willets K A and Van Duyne R P 2007 Localized surface plasmon resonance spectroscopy and sensing Annu. Rev. Phys. Chem. 58 267-97

[42] Righini M, Zelenina A S, Girard C and Quidant R 2007 Parallel and selective trapping in a patterned plasmonic landscape Nat. Phys. 3 477-80

[43] Sow I, Grand J, Lévi G, Aubard J, Félidj N, Tinguely J C, Hohenau A and Krenn J R 2013 Revisiting surfaceenhanced raman scattering on realistic lithographic gold nanostripes J. Phys. Chem. C 117 25650-8

[44] Craighead H G 2000 Nanoelectromechanical systems Science 290 1532-5 